\title{
Precision Medicine, Personalized Medicine, Omics and Big Data: Concepts and Relationships
}

\section{Xiaohua Douglas Zhang*}

Biometrics Research, WP53B-120, Merck Research Laboratories, P.O. Box 4, West Point, PA 19486, USA

On January 20, 2015, US President Obama announced at his 2015 State of the Union Address that he was launching a new precision medicine initiative [1]. On January 30, the Obama administration unveiled details about the Precision Medicine Initiative. Launched with a \$215 million investment in the US President's 2016 budget, the Precision Medicine Initiative will pioneer a new model of patientpowered research that ultimately help deliver the right treatment to the right patient at the right time [2]. On March 11, 2015, it had been reported that China is planning to invest 60 billion Yuan (nearly $\$ 10$ billion) in precision medicine ( 20 billion from the Central Government and the remaining 40 billion from local governments and companies) before 2030 [3].

So, what is precision medicine? How is it related to other terms such as personalized medicine and omics (especially Pharmacogenomics and Pharmacoproteomics)? In this article, I elaborate the concepts and their relationships.

\section{Precision Medicine}

Precision medicine has been thought to revolutionize how we improve health and treat disease. Today, most medical treatments are designed for the average patient using the "one-size-fits-all" approach. However, in many cases, this approach isn't effective because treatments can be very successful for some patients but not for others. Precision medicine is a field of medicine that takes into account individual differences in people's genes, microbiomes, environments, family history, and lifestyles to make diagnostic and therapeutic strategies precisely tailored to individual patients.

Precision medicine is a newer term referring to a similar field compared to another term "personalized medicine". The term 'precision medicine' entered the scientific lexicon in 2008 when business strategist Clayton Christensen, of Harvard Business School in Boston, coined the expression to describe how molecular diagnostics allows physicians to unambiguously diagnose the cause of a disease without having to rely on intuition [4]. The name didn't gain traction until 2011 when a committee convened by the US National Research Council laid out a blueprint for modernizing the taxonomy of disease on the basis of molecular information such as causal genetic variants, rather than a symptom-based classification system. They called the report Toward Precision Medicine [4].

\section{Personalized Medicine}

The term, 'personalized medicine' first appeared in published works in 1999 with the creation of some of the field's core concepts even dating back to the early 1960s [5]. In short, personalized medicine simply means selection of treatment best suited for an individual. Originally, personalized medicine is the concept that selection of a treatment should be tailored according to the individual patient's specific characteristics, including age, gender, height/weight, ethnicity, diet, and environment, versus a decision based on 'standards of care' derived by averaging responses across large cohorts of individuals in clinical trials [6].
Personalized medicine came to the attention of scientists when they began to realize the promise of the Human Genome Project. The scientists announced they had deciphered the human genome in 2000 and the Human Genome Project was completed in 2003. By lying out in order the 3.2 billion units of our DNA, researchers sparked a firestorm of discovery and an explosion of genomic knowledge. Accompanied with them are the rapidly emerging novel omics technologies including microarrays, whole-genome single nucleotide polymorphism [SNP] chips, RNA interference high-throughput screening, next-generation sequencing etc. All these launch a new era, the so-called genomic revolution era which offers us boundless potential and great promise including the development of personalized medical products for each individual based on his/her unique genomic information [7,8]. With the advance of genomics science and the emerging of new omics technologies, personalized medicine is now commonly defined as a combination of molecular profiling (omics methods) and traditional methods, such as family history, environment and lifestyle, to create diagnostic and therapeutic strategies precisely tailored to individual patients [5-7].

\section{Precision Medicine and Personalized Medicine}

Some scientists began to consider changing the name from personalized medicine to precision medicine after the US National Research Council published their report named "Toward Precision Medicine: Building a Knowledge Network for Biomedical Research and a New Taxonomy of Disease" in 2011 [8]. One of the common complaints about the term 'personalized medicine' is as follows. "First of all, 'personalized' implies the prospect of devising a different treatment for each individual patient. On one hand, that's how medicine is practiced every day, and on the other, it's a complete fallacy." [4]. Thus, some researchers began to retire the phrase "personalized medicine" and replace it with "precision medicine" with the idea that molecular information improves the precision with which patients are categorized and treated [4]. However, not everyone supports renaming personalized medicine. In addition, many people believe that personalized medicine and precision medicine are not all the same. As a result, both terms exist nowadays.

There are differences between precision medicine and personalized medicine. In theory, first, precision medicine focuses on classifying individuals into subpopulations that differ in their susceptibility to a particular disease, in the biology and/or prognosis of those diseases

*Corresponding author: Xiaohua Douglas Zhang, Biometrics Research, WP53B-120, Merck Research Laboratories, P.O. Box 4, West Point, PA 19486, USA, Tel: 215-6520522; Email: xiaohua_zhang@merck.com

Received April 16, 2015; Accepted April 17, 2015; Published April 23, 2015

Citation: Zhang XD (2015) Precision Medicine, Personalized Medicine, Omics and Big Data: Concepts and Relationships. J Pharmacogenomics Pharmacoproteomics 6: e144. doi:10.4172/2153-0645.1000e144

Copyright: (c) 2015 Zhang XD. This is an open-access article distributed under the terms of the Creative Commons Attribution License, which permits unrestricted use, distribution, and reproduction in any medium, provided the original author and source are credited. 
they may develop, or in their response to a specific treatment [9]. Hence, precision medicine focuses on patient subpopulations, one step-up from the individual focus of personalized medicine. Second, in terms of pharmaceutical and medical research, precision medicine is an approach to integrating clinical and molecular information to understand the biological basis of disease and accordingly to discovering and developing medical products that deliver superior outcomes for patients. Thus, precision medicine focuses on the research and development paradigm that will help enable more patient-centered clinical practice, including (as in personalized medicine) treatment decision-making based on genetic and other related information of an individual. Thus, precision medicine is more research-oriented whereas personalized medicine is more clinical practice-oriented.

In practice, the use of the term "precision medicine" can extend beyond treatment selection to also cover creating unique medical products for particular individuals. Similarly, although the term 'personalized medicine' suggests that decisions on preventive, diagnostic, or therapeutic strategies are tailored to the characteristics of an individual, in practice, personalized medicine usually refers to the stratification of apparently clinically homogeneous patients into subpopulations with distinct clinical prognosis or response to treatment [10]. Hence, precision medicine and personalized medicine have so much overlap that they are often used interchangeably in practice.

\section{Precision Medicine and Omics}

The advance of precision/personalized medicine heavily relies on the ability to study biological phenomena at omics levels although the practice of precision/personalized medicine does not use only omics data and knowledge. This is because molecular characteristics obtained from omics data can classify diseases and identify subpopulation of patients suitable to certain common treatment more precisely.

Traditionally, biology focuses on descriptions of biological phenomena and mechanisms. Nowadays, biology has become an increasingly data-rich subject. Following this trend, many of the emerging fields of large-scale data-rich biology are designated by adding the suffix '-omics' onto previously used terms. Specifically, the word omics refers to a field of study in biology ending in the suffix omics and its related ome addresses the objects of study of such a field. For example, a genome is the sum total of all an individual organism's genes whereas genomics is the study of the genomes of organisms.

Pharmacogenomics is the study of how a person's response to drugs is affected by his/her genetic makeup. It combines pharmacology (the science of drugs) and genomics (the study of genes and their functions) to develop effective, safe medications and doses that will be tailored to a person's genetic makeup. Pharmacoproteomics, essentially a sub-discipline of functional pharmacogenomics, is a study of how the protein content of a cell or tissue changes qualitatively and quantitatively in response to treatment or disease, what the proteinprotein and protein-ligand interactions are in related to drug response, and how a person's protein variants in quality and quantity affect a person's response to a drug. Currently, the pharmaceutical industry has become very interested in Pharmacoproteomics with the expectation that this technology will lead to the identification and validation of protein targets and, ultimately, to the discovery and development of viable drug candidates. Pharmacogenomics and Pharmacoproteomics will help the prescription of drug and related doses to a patients based on his/her response to a drug, thus greatly promoting the advance and practice of precision/personalized medicine.

\section{Precision Medicine and Big Data}

In 2012, the Obama administration announced the Big Data Research and Development Initiative [11], which explored how big data could be used to address important problems faced by the government. Since then, Big Data becomes such a big term that people tend to claim any kind of data analysis to be "Big Data" analysis. There are various definitions for Big Data. One definition is, "Big data is a blanket term for any collection of data sets so large and complex that it becomes difficult to process using on-hand database management tools or traditional data processing applications." [12]. It has the "4V's+1" characteristics, namely Volume (i.e., large data size), Velocity (i.e. high speed of change), Variety (i.e., various forms of data sources), Veracity (i.e., uncertainty of data) and Driving Results (i.e., generating value).

Omics data, mobile internet real-time data and electronic health record data are the top three areas for Big Data in medical research. Precision medicine will use all of these three Big Data. In fact, among the $\$ 215$ million investment in the USA President's 2016 Budget, $\$ 130$ million (over $60 \%$ ) will be used for building a large US cohort for precision research [13]. In this cohort study, the scientists will use extensive omics data, electronic health record data and mobile internet data [14]. Thus, omics and medical big data are one of the keys in the success of precision medicine.

\section{References}

1. https://www.whitehouse.gov/blog/2015/01/21/precision-medicine-improvinghealth-and-treating-disease

2. https://www.whitehouse.gov/the-press-office/2015/01/30/fact-sheet-presidentobama-s-precision-medicine-initiative

3. http://finance.china.com.cn/industry/medicine/yyyw/20150326/3024172.shtml

4. Katsnelson A (2013) Momentum grows to make 'personalized' medicine more 'precise'. Nature Medicine 19: 249.

5. Jain KK (2002) Personalized medicine. Curr Opin Mol Ther 4: 548-558.

6. Jain, Kewal K (2009) Textbook of Personalized Medicine. Springer Science+Business Media LLC, Springer, New York.

7. Collins FS, Green ED, Guttmacher AE, Guyer MS (2003) A vision for the future of genomics research. Nature 422: 835-847.

8. Zhang XHD (2011) Optimal High-Throughput Screening: Practical Experimenta Design and Data Analysis for Genome-scale RNAi Research. Cambridge University Press, Cambridge, UK.

9. The US National Research Council Committee on A Framework for Developing a New Taxonomy of Disease (2011) Toward Precision Medicine: Building a Knowledge Network for Biomedical Research and a New Taxonomy of Disease. US National Academies Press, Washington DC.

10. Völzke H, Schmidt CO, Baumeister SE, Ittermann T, Fung G et al. (2013) Personalized cardiovascular medicine: concepts and methodological considerations. Nature Review Cardiology 10: 308-316.

11. https://www.whitehouse.gov/sites/default/files/microsites/ostp/big_data_ press_release_final_2.pdf

12. http://en.wikipedia.org/wiki/Big_data

13. Collins FS (2015) Vision for the cohort and the precision medicine initiative. NIH Workshop on Building a Precision Medicine Research Cohort, Bethesda Maryland, USA.

14. Work Group for Building a Consortium of Cohorts (2015) Precision medicine: building a large U.S. research cohort. NIH Workshop on Building a Precision Medicine Research Cohort, Bethesda, Maryland, USA. 